\title{
Characterization of Hydroxyapatite by Electron Microscopy
}

J. Sánchez Hernández ${ }^{*}$, V. Rodríguez Lugo ${ }^{* *}$ and M. J. Arellano Jiménez ${ }^{* * *}$

* Instituto de Física Luis Rivera Terrazas. Benemérita Universidad Autónoma de Puebla. 4 sur 104 72000 Puebla, Pue.

** Centro Universitario de Vinculación. Benemérita Universidad Autónoma de Puebla. 29 Oriente 601-1 Ladrillera de Benítez 72540 Puebla, Pue.

${ }^{* * *}$ Instituto de Física, UNAM. Apartado postal 20364, 01000. México D.F.

Bones and teeth of vertebrate animals are constituted by a mineral phase very similar to hydroxyapatite $\left(\mathrm{Ca}_{10}\left(\mathrm{PO}_{4}\right)_{6}(\mathrm{OH})_{2}\right)$, for this reason, this material receives a great attention in the industrial and biological area. The structure and particular properties of the synthetic hydroxyapatite used in the production of artificial bones and teeth are very sensitive to the conditions of synthesis, for which, the studies carried out on the methods of synthesis of hydroxyapatite are very important because they determine the growth of the crystal, morphology, aggregation, nucleation and dissolution properties of hydroxyapatite. The present work was developed in order to establish the effects of reaction time in morphological and structural characteristics of hydroxyapatite.

The synthesis was carried out starting of brushite and starfish, the last one like source of calcium carbonate, under hydrothermal conditions using stoichiometric amounts of both reagents, varying times of reaction and maintaining pressure and temperature constant. The products of reaction were characterized by X-ray diffraction (XRD), scanning electron microscopy (SEM) and transmission electron microscopy (TEM).

The growth of grouping crystals in form of fibers of the order of $1 \mu \mathrm{m}$ to $370 \mu \mathrm{m}$ long and $0.15 \mu \mathrm{m}$ to $10 \mu \mathrm{m}$ diameter with a composition of $\mathrm{C}, \mathrm{O}, \mathrm{P}$ and $\mathrm{Ca}$ was observed (Figure 1). It is possible also observe the growth of fibers of the order of $5 \mu \mathrm{m}$ of longitude and the same composition that last. Besides particles of the order of $3 \mu \mathrm{m}$ to $10 \mu \mathrm{m}$ with an elementary composition of $\mathrm{C}, \mathrm{O}, \mathrm{P} \mathrm{Mg}$ and $\mathrm{Ca}$ and prisms of the order of $17 \mu \mathrm{m}$ to $43 \mu \mathrm{m}$ were also observed.

By TEM, fibers with a size of $800 \mathrm{~nm}$ long and $160 \mathrm{~nm}$ diameter were observed and they show a growth in groups (Figure 2a). Each fiber exhibits the typical hexagonal structure of the hydroxyapatite. The diffraction pattern (Figure $2 \mathrm{~b}$ ) corresponds to hydroxyapatite fibers according to the indexation.

\section{References}

[1] Joon Bu Park. Biomaterials Science and Engineering. Plenum Press. N.Y. 1984

[2] D. B. Williams and C. B. Carter. Transmission Electron Microscopy. A Text Book for Materials Science. Plenum Press. N. Y. 1996

[3] V. Rodríguez-Lugo, G. A Camacho-Bragado and V. M. Castaño, Materials and Manufacturing Processes, In Press (2002) 


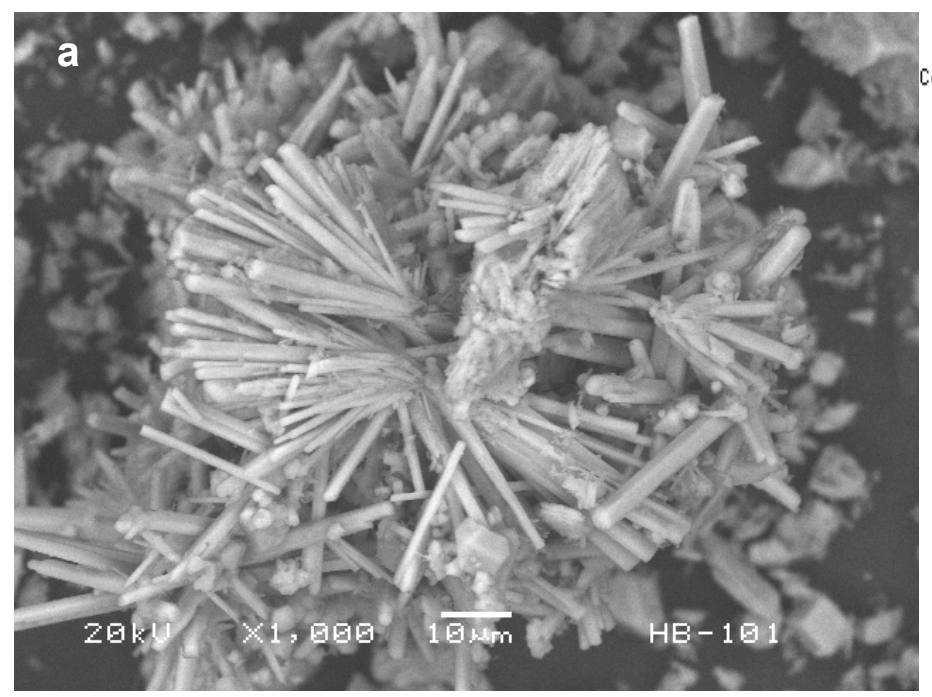

b

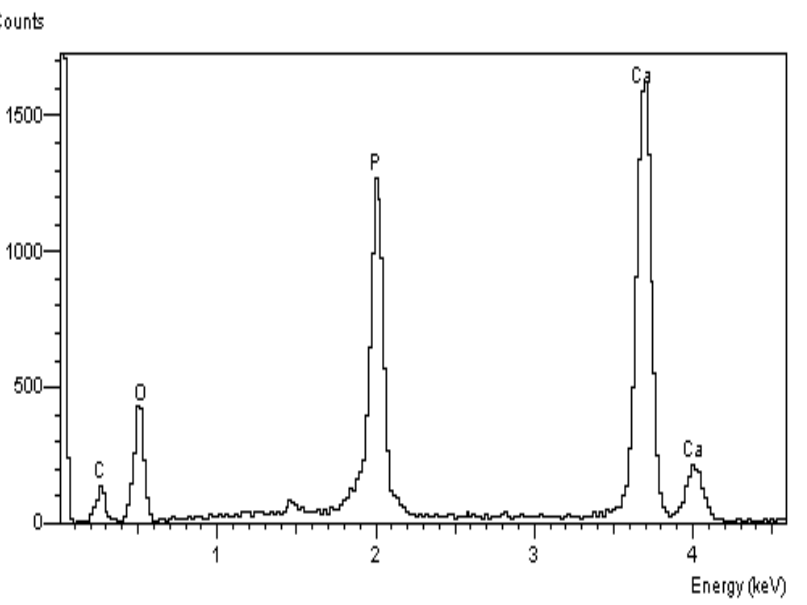

Figure 1. a) Hydroxyapatite fibers in order about $30 \mu \mathrm{m}$. b) EDS spectrum of hydroxyapatite fibers.

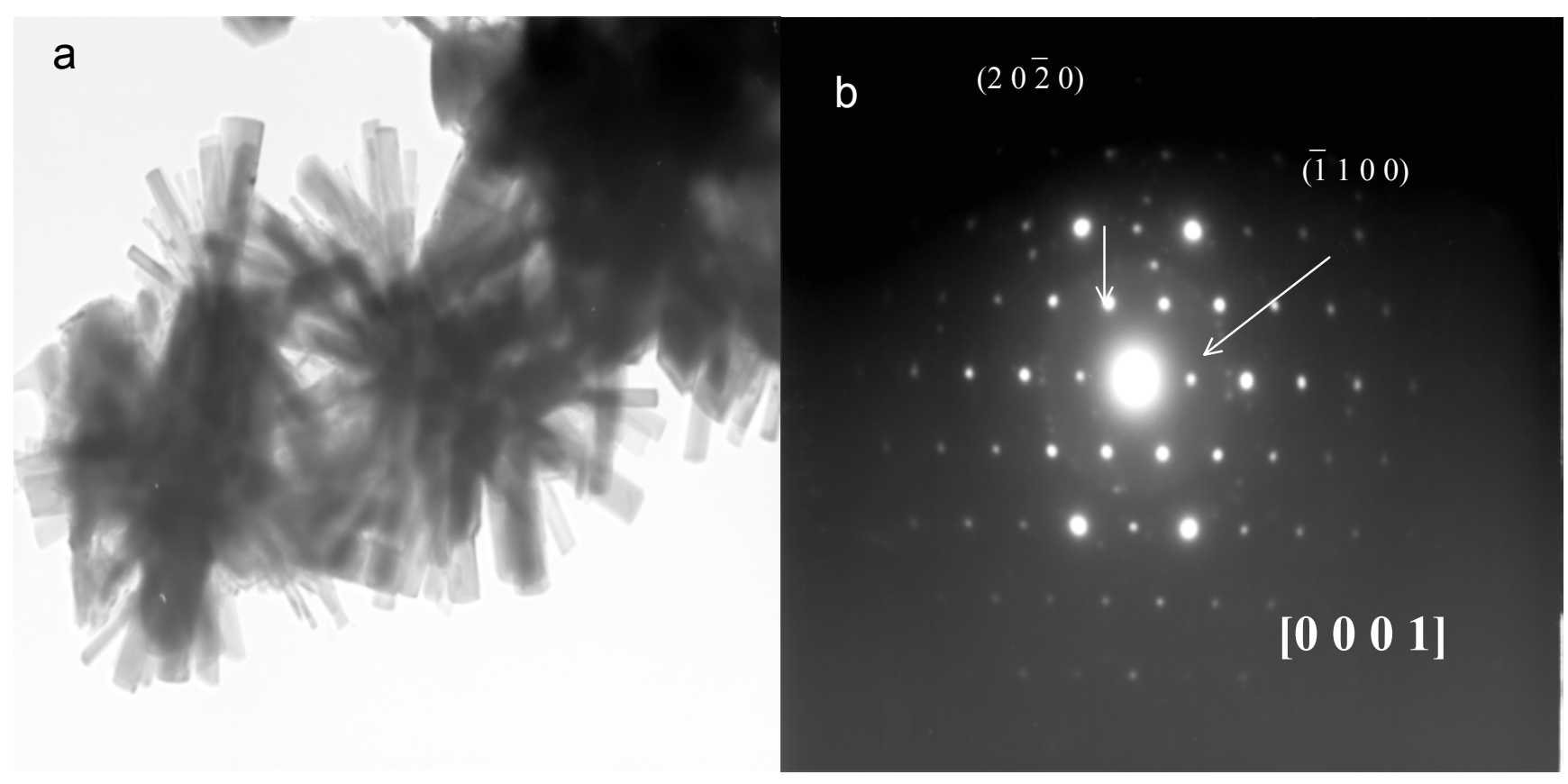

Figure 2. a) Bright file image of hydroxyapatite fibers forming a group in order to $800 \mathrm{~nm}$ long and $160 \mathrm{~nm}$ diameter. b) Diffraction pattern in [0 $\left.\begin{array}{llll}0 & 0 & 0 & 1\end{array}\right]$ direction. 